\title{
Function-Oriented Business Process Improvement Framework for Customer Relationship Management Section in Large Scale Organization
}

\author{
Suprangtip Poonun, Sotarat Thammaboosadee, and Supaporn Kiattisin
}

\begin{abstract}
Customer Relationship Management (CRM) is critical and essential to such organization, especially to the large scale organization since its involved customers may be covered to the people, citizen, organization or government sections. Anyway, according to the nature and culture of the traditional job design, their business processes are costly and inconsistent because of their actor (or department) oriented design which leads to the difficulties in improvements. This paper proposes an idea of improving the business processes, based on Business Process Improvement (BPI) concept in function-oriented, to solve the existing work problems and suggest the possible solution for the future to achieve the organization goal. The evaluation was done by both the officer and the executive.
\end{abstract}

Index Terms-Function-oriented, business process improvement, customer relationship management.

\section{INTRODUCTION}

Business Process Improvement (BPI) [1] is a method of designing organizational adjustment which continues to increase operational efficiency by focusing on improving the business taken from analysis of the problem or finding of the real cause. An intermediate information collecting is used in the process of adjusting in current work or current data model into the new one. It also minimizes the loss of jobs that is worthless in the organization and increases yield of products and services. There are some factors involved in this consideration such as the inability of customers' demands response, high operating costs, operational delays, personnel lack of knowledge and expertise, etc. Additionally, there are some external factors such as high competition, increasing in competitors, changing in economic conditions, new technology, and the requirements of the client and the organization. Thus, improving of business processes is important [1] because the improvements aim to simplify the procedure for non-critical tasks. The processes with the same or strongly similar procedures are combined into a single one, rated by comparative rules with updating methods of working to be more convenient such as a one-stop service. Technology usage assists in managing ongoing operational to be less time consuming. Saving resources in managing, e.g. personnel, cost, time, and equipment management, operates in

Manuscript received April 27, 2015; revised August 21, 2015.

Suprangtip Poonun, Sotarat Thammaboosadee, and Supaporn Kiattisin are with Technology of Information System Management Division, Faculty of Engineering, Mahidol University, Nakhon Pathom, 73170, Thailand (email: i.neayz@msn.com, sotarat.tha@mahidol.ac.th, supaporn.kit@mahidol.ac.th). environments that change. According to the Business Process Improvement (BPI), there are many agencies use the customer relationship management involves their works.

Customer Relationship Management (CRM) [2] in most of organizations recognizes the importance of developing a client relationship management system to help in managing of customer database belonging to the agency for the purpose of management of the project in the basis of the mission in such agency. Monitoring and evaluation of the analytical data is reported which makes the information is accurately accessible. Therefore, the CRM is managed by various involved agencies. For example, the system could be the centre system, customer service system, marketing system, sales system, etc.

In the present, most organizations have a pursuit profit but they are also has internal problems, one of those is characterized by a non-profit selling products and services, mostly with government support. Additional factors are [3], for example, making less sales management, including complicated steps in the sale process, building a barrier in the workplace, requiring documents are in multiple formats, long duration working, personal confusing, and lacking of understanding in job roles and responsibilities. Therefore, BPI, coupled with the development of customer relationship management system, is an interest domain to be applied. Additionally, some of CRM driven organizations designed the current process flows based on the pre-defined and established section complied with an organization structure. Each business process is bound with specific section (or department). This designing methodology is costly and inconsistent because their actor-oriented design may leads to the difficulties in improvements.

This paper proposes a designing framework for Business Process Improvement (BPI) in customer relationship management systems (CRM) by function-oriented approach and also its evaluation. A case study example is an e-government company with a mission to develop a comprehensive operational, management of infrastructure, to survey, to research, and to analyze problems and success factors. These missions involve the consultation services, technical support and training to enhance the skills, knowledge and the dissemination of information related under the partnership with government agencies and the private sector by considering covers three aspects: economic, social, and environmental, for examples, budget savings, decrease the operations of government, promote interaction between government and citizens.

The rest of this paper is organized as follows. In Section II, we describe a concept and features of CRM. Section III 
presents the general concepts of BPI. Section IV describes the proposed framework and demonstrated example case study. Consequently, the evaluation, discussion and conclusion are described in latter sections respectively.

\section{Customer RElationship MANAGEMENT (CRM)}

\section{A. Concepts and Principles}

The CRM is consisted of some concepts including: learning about ongoing customer, retaining the customers, satisfying the demands of customers and offer to fulfil them, dealing with different customers, and personally interacting with customers. Building customer relationships increases value for both clients and organization which is such a compelling strategy. When the CRM is executed properly, the focus on building relationships and brand loyalty is a "win-win" for customers and the organization [4]. This strategy takes a holistic view of customers and consolidates information from across the organization, regardless of geography, department, function, contact channel, social community, or product line. It usually focuses on revenue and retention more than on reducing costs. It also enables information sharing and interaction across the organization and creates business rules to drive all customer relationship management decisions and automation. Consequently the effective management of customer relationships is a way of doing business, not just a technology project which is only an enabler [5]. The objectives of the CRM [6], [7] could be included identifying new opportunities, reducing missed opportunities reducing customer defection, improving customer service, enhancing appearance of organization, storing of organization information, and reinventing marketing.

\section{B. Feature of CRM}

The key features of a CRM system, as demonstrated in Fig. 1, which deliver positive customer experience are usability, flexibility, high performance, and scalability [8]. CRM should be flexible enough to stay in touch with different users, reflect different requirements in different industries and be accessible to external or internal users. Furthermore, CRM should work over any communication channel and must integrate other systems to provide a single view of, and for the users [9].

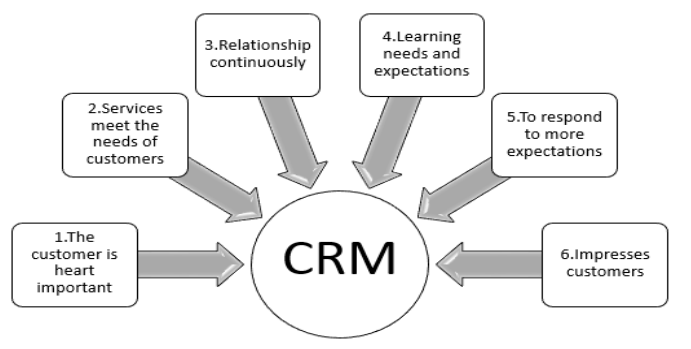

Fig. 1. Feature of CRM.

\section{Customer Relationship MANAGEMENT (CRM)}

\section{A. Concepts and Principles}

As shown in Fig. 2, the general procedures of BPI [10] could be divided in phases as follow:
Scope Phase - The scope of the project in terms of time, costs, resource estimate includes an understanding of stakeholders and customers.

Document and Analyze phase - This phase refers to documenting the implementation and analysis of current conditions. Disclosure of operation should be revised, to identify roles and responsibilities within the organization with understanding.

Redesign Phase - To gear to the goals and objectives of the new design have to simulate and evaluate for the project management, monitoring, integration entail, and analyzing the terms and costs.

Implement Phase - This phase is to define scope and the procedure that have been approved for compliance with the appropriate procedures to implement process improvements.

Operate Phase - The operate phase represents the day-to-day management of the new or redesigned process [11]. To ensure the operation, the procedures are followed: steps in the preparation of the audit plan, data collection and establish a monitoring process to monitor, analysis, and finally a conclusion [12], [13].

Review and Evaluate Phase - This phase is to monitor and evaluate period into improvement by identifying and complying the indicators in the assessment against goals and objectives. This will enable stakeholders and process owners to decide whether to continue or to improve further.

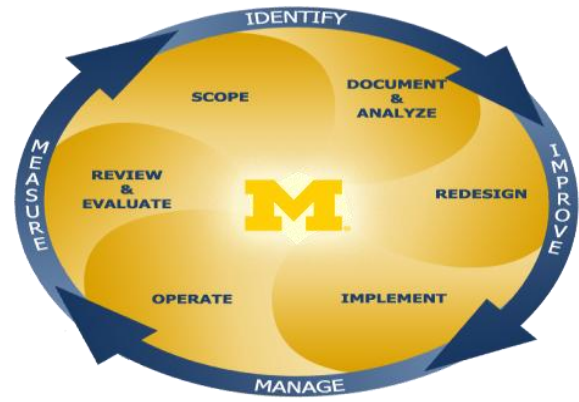

Fig. 2. Business process improvement methodology.

\section{FUNCTION-ORIENTED BPI FRAMEWORK}

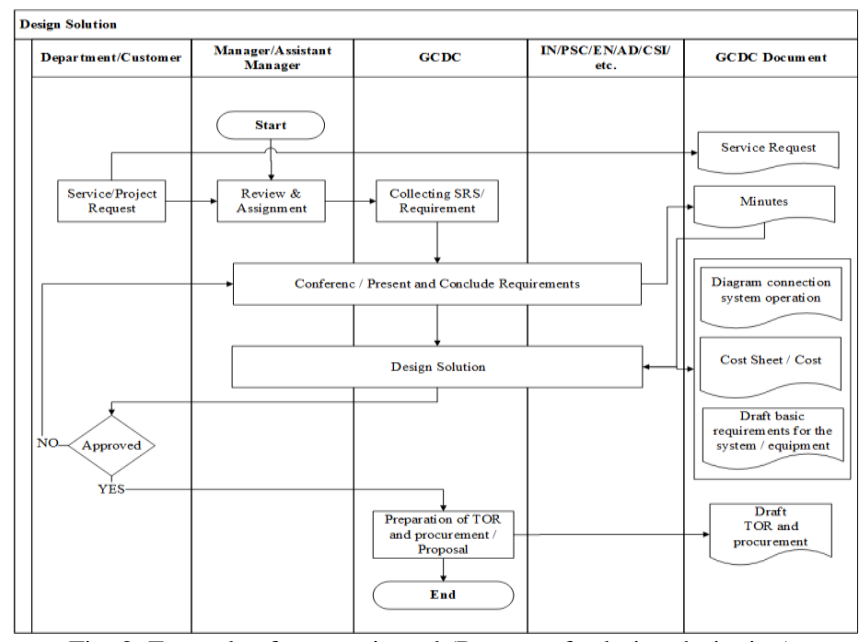

Fig. 3. Example of actor-oriented (Process of solution designing).

This paper proposes a framework of applying the function-oriented Business Process Improvement (BPI) with the agency on customer relationship management sections, 
scoped to the sale management system as case study. The function-oriented BPI refers the function-oriented BPI refers to processes or data flow is an improvement of the traditional actor-oriented business process. The function oriented BPI is represented in the form of a process arrow chart of the work conveys. Workflows hierarchically until the end of the process Flow of work to implement a swim-lane diagram have input data, output and functions relate work to the clearly, but the actor-oriented is the traditional model as shown in Fig. 3. It has the disadvantage of having a beginning and an ending are not connected to the work that occurs in the process of implementation and cannot be seen an overview a processes and sub-processes will shows only the tasks and workflows. An overview of the CRM in the selected organization is shown in Fig. 4.

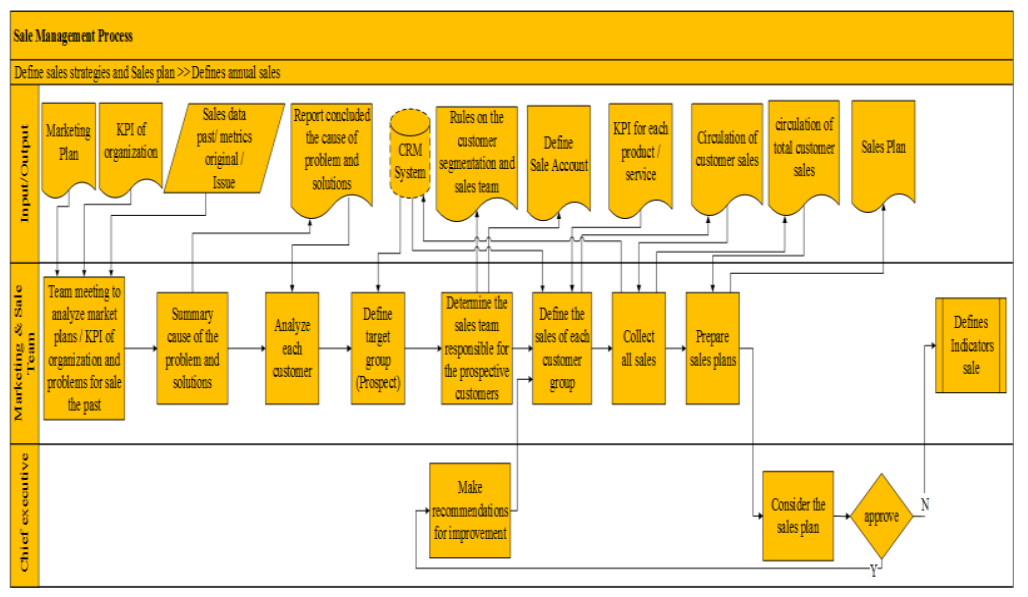

Fig. 6. Swim-lane diagram of defines annual sale sub-process.

Therefore, the study has to clarify the source, the review process, and the structure of the organization for preparing of the business process and business flowchart. Analyses the design process and the forms that must be used in each process are as follows:

Step 1: Project Plan - Schedule of meetings, interviews, and workshops.

Step 2: Study current Status of the organization and requirements using policies, goals, vision, strategy, mission, operational processes, structure and functions of the organization, products and services, and other related operations. This step is to comply with the implementation of the new customer relationship management, named as "As-Is Analysis" which consists of guidelines for the implementation project management, data collection and needs, development of architectural projects, commercial development, business intelligence development, web portal development and linkage to other systems.

Step 3: Analyze the operational goals of the organization (business goals) and currently core business process and provide the suggestions to improve the process by comparing with international standards. Then, Prepare and present operational process redesign (To-Be Process) by the mapping process (Business Flowchart) in accordance with the policy and vision. The compliance with various standards and opportunities to improve processes to increase operational efficiency by providing a form that used in the process of CRM systems including assign responsibility for each process.

Step 4: The preparation of a To-Be process form have to be presented to the responsible parties within the organization to consider the comments and updates, but the new designed process must be trained for practitioners within the organization.

Step 5: Track execution, evaluate, and recommend how to improve customer relationship management in the operation of the organization.

Consequently, this part shows how to design function-oriented BPI to a CRM sections for a sales management process, for example, in type agencies has to pursuit of profit and nonprofit of sale, as shown in Fig. 5. The arrow chart diagram shown in this Figure was designed by the concept of function-oriented which emphasizes on the main process and its sub-processes and slightly neglects the actor (or department) that involved to the jobs. This concept typically shows the more obvious perspective of the selected process.

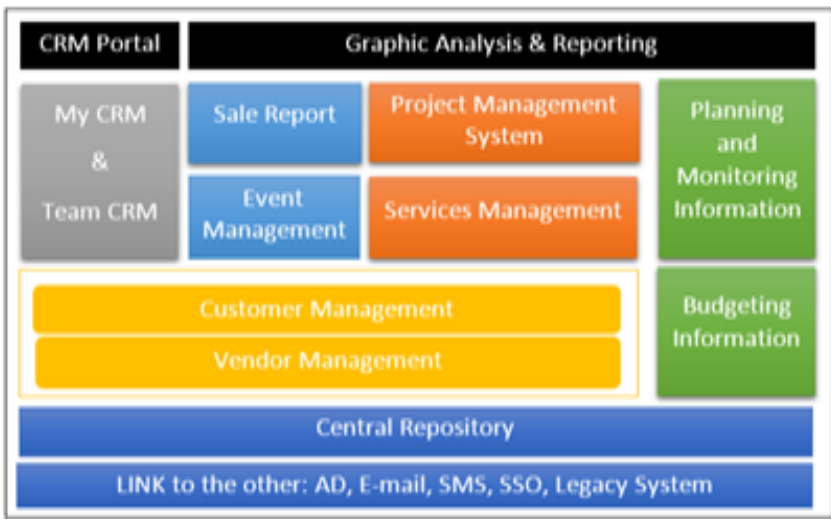

Fig. 4. Customer relationship management system overview.

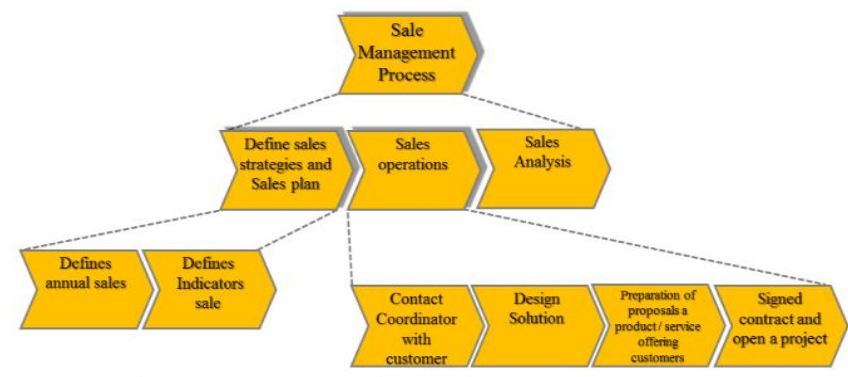

Fig. 5. Process arrow chart (Sale management process). 
TABLE I: TYPE SIZES FOR CAMERA-READY PAPERS

\begin{tabular}{|l|l|l|l|}
\hline GUIDELINE & Low & Medium & High \\
\hline 1. Decrease the time in operation & & I/E & \\
\hline 2. Know Role Responsibilities & & & I/E \\
\hline 3. Support with Standard & & & I/E \\
\hline 4. Consistency on Technology & I & E & \\
\hline 5. Linked data is accurate & & I & E \\
\hline $\begin{array}{l}\text { 6. Assist in the planning and } \\
\text { decision }\end{array}$ & & I/E & \\
\hline 7. Reduce costs & & $\mathrm{I}$ & $\mathrm{E}$ \\
\hline $\begin{array}{l}\text { 8. Increase organizational } \\
\text { efficiency }\end{array}$ & & & $\mathrm{I} / \mathrm{E}$ \\
\hline 9. Achieve the goals organization & & $\mathrm{I}$ & $\mathrm{E}$ \\
\hline $\begin{array}{l}\text { 10. Customer satisfaction in our } \\
\text { products and services. }\end{array}$ & & & $\mathrm{I} / \mathrm{E}$ \\
\hline 11. Better restructuring operation & & $\mathrm{E}$ & $\mathrm{I}$ \\
\hline $\begin{array}{l}\text { 12. Recognized within } \\
\text { organization }\end{array}$ & $\mathrm{E}$ & $\mathrm{I}$ & \\
\hline
\end{tabular}

\section{EVALUATION AND DISCUSSION}

According to the evaluation results shown in Table I, the comparative satisfaction between the traditional actor-oriented process and the proposed function-oriented method collected from the Chief Information Officer (CIO) and the Chief Executive Officer (CEO) have commented on the new designed CRM business process is superior to the traditional one based on the guidelines defined in scope best practice. In Table I, the symbol 'I' and 'E' refer to the opinion of the $\mathrm{CIO}$ and $\mathrm{CEO}$ respectively.

According to an assessment of satisfaction collected by CEO and CIO with the scoring scale: Low-Medium-High (1-2-3), an average score is $2.42 / 3.00$ point. A recognizing within an organization is difficult individuals within an organization to accept the changes made. However, the rest parts are in quite high satisfaction because the proposed method provides a great impact on the organization in all aspects of improving.

\section{CONCLUSIONS}

Customer Relationship Management (CRM) is required to improve the process because it is the core business process that can propel the organization into the future. We proposed a framework to improve the actor-oriented business process to be a function-oriented business flow. The improvement of business processes in customer relationship management is a new model called function-oriented, can help organizations responding to customer satisfaction which could lead to lower costs, view the overall work process, a clear division of responsibility, deploy the technology is better and recognized in international standards even more. This framework is confirmed by $\mathrm{CEO}$ and $\mathrm{CIO}$ of the organization with the high satisfaction levels.

\section{REFERENCES}

[1] T. N. Damij, "Improvement of a clinical business process," in Proc. Computer Modeling and Simulation, Sept. 8-10, 2008, pp. 305-310.

[2] H. Bing et al., "Design and implementation of customer relationship management system based on structured object-oriented methodology," in Proc. 2011 International Conference on Information Technology, Computer Engineering and Management Sciences (ICM), vol. 3, pp. 24-25, Sept. 2011, pp. 390, 393.

[3] G. K. Agrawal and B. Daniel, "The development of services in customer relationship management (CRM) environment from technology perspective," J. Service Science \& Management, no. 2, pp. 432-438, Aug. 21, 2009.

[4] B. Cleveland, "The 12 principles for building profitable customer relationship," 2014.

[5] H. Beyadar and K. Gardali, "The study of customer relationship management method," in Proc. 2011 5th International Conference on Application of Information and Communication Technologies (AICT), Oct. 12-14, 2011, pp. 1-4.

[6] M. Sarafraz and Memarzade, "The paradigm of electronic customer relationship management (ECRM) in project management, customer retention," presented at the First International Conference of Electronic Banking, Tehran, 2007.

[7] T. A. Feizbakhsh, F. Saeed, T. Alireza, V. Reza, and K. Saed, "Theoretical model of customer relationship management in organizations," International Journal of Business and Behavioral Sciences, vol. 3, no. 11, pp. 63-70, Nov. 2013.

[8] B. Francis, "Customer relationship management: Concepts and technologies," 2009.

[9] A. Vieira and S. Figueiredo, "Using a CRM approach for implementing an information system to support ITIL," in Proc. 2012 Eighth International Conference on the Quality of Information and Communications Technology (QUATIC), Sept. 3-6, 2012, pp. 178, 181.

[10] S. Andrea, "ITS process improvement methodology (Methodology overview)," Nov. 2010.

[11] ITS University of Michigan "Methodology: Process improvement," 2013.

[12] R. Brandao and M Wynn, "Product lifecycle management systems and business process improvement - A report on case study research," in Proc. the Third International Multi-Conference on Computing in the Global Information Technology, July 27, 2008-Aug. 1, 2008, pp. 113-118.

[13] S. Chen, B. Mulgrew, and P. M. Grant, "A clustering technique for digital communications channel equalization using radial basis function networks," IEEE Trans. on Neural Networks, vol. 4, pp. 570-578, July 1993.

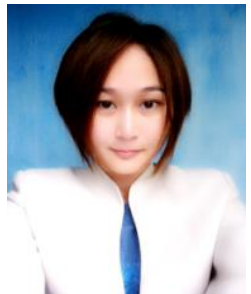

Suprangtip Poonun received the B.B.A in business information technology management of information technology from Rajamangala University of Technology Rattanakosin, Nakhonpathom, Thailand, in 2013 and received the M.Sc. degree in information technology management from Mahidol University, Nakhonpathom, Thailand, in 2015, where she is currently pursuing the Ph.D. degree. Her current research interests include business process improvement and customer relationship management.

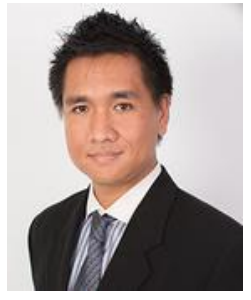

Sotarat Thammaboosadee was born in 1982 and received his B.Eng. and M.Sc. degrees in computer engineering and technology of information system management from Mahidol University, Thailand, in 2003 and 2005 respectively. He also received a Ph.D. in information technology from King Mongkut's University Technology Thonburi, Thailand, in 2013 $\mathrm{He}$ is now a lecturer at Technology of Information System Management Division at Faculty of Engineering, Mahidol University, Thailand. His research interests include data mining in several domains such as the legal domain, healthcare domain and management domain. He also interests the research filed of technology valuation and business process improvement in CRM section.

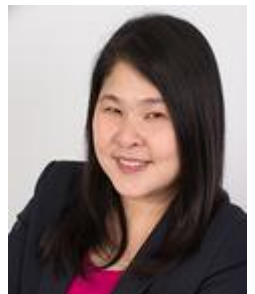

Supaporn Kiattisin received her B.Eng. and M.Eng. degrees in computer engineering and electrical engineering from Chiang Mai University, Thailand, in 1996 and King Mongkut's University Technology Thonburi, Thailand, in 2000 respectively. She also received a Ph.D. in electrical and computer engineering from King Mongkut's University Technology Thonburi, Thailand, in 2007. She is now a vice-director at Technology of Information System Management Division and program chair of Information Technology Management program at Faculty of Engineering, Mahidol University, Thailand. Her research interests include enterprise architecture, risk management, business process improvement, customer relationship management, and human resource management 